\title{
Guillermo de Ockham y el desafío escéptico en los Quodlibeta Septem
}

\author{
DENI GAMBOA \\ Universidad de Québec en Montreal \\ denigalo@gmail.com
}

\begin{abstract}
Resumen: La epistemología de Guillermo de Ockham expuesta en los Quodlibeta Septem conduce a conclusiones escépticas. La proposición anterior sintetiza la hipótesis de este ensayo. La demostración que aquí expongo está basada en mi propuesta de interpretación sobre el modo en que se desarrolla todo proceso cognoscitivo de singulares. Tal interpretación puede expresarse en dos proposiciones: 1) el conocimiento intuitivo y el conocimiento abstractivo son formas distintas de un asentimiento simple; 2) Ockham, dentro de su propio esquema epistemológico, intenta evitar conclusiones escépticas por medio de la noción de conocimiento reflexivo. Esta última noción, sin embargo, no nos permitiría justificar que nuestras creencias sean verdaderas.
\end{abstract}

Palabras clave: conocimiento intuitivo, conocimiento abstractivo, conocimiento reflexivo

\begin{abstract}
After analyzing the Quodlibeta Septem it is possible to state that Ockham's epistemology leads to skeptical conclusions. This statement is grounded on my interpretation of the way in which any cognition process of singular entities develops. My interpretation might be expressed in two propositions: 1) the intuitive and abstractive cognitions are different forms of a simple assent, and 2) within his own espistemological system, Ockham tries to avoid skeptical conclusions by way of reflexive cognitions. Nevertheless, the idea of reflexive cognitions would not let us justify which thoughts are true. In Ockham's epistemology, the idea of reflexive cognitions does not solve the skeptical challenge.
\end{abstract}

Key words: intuitive cognition, abstractive cognition, reflexive cognition

Desde la primera mitad de siglo xx, la epistemología de Guillermo de Ockham ha sido interpretada como una teoría que deriva en conclusiones escépticas. Tal interpretación fue puesta en duda desde la década de 1940. Podemos hallar el fundamento de tal discrepancia en el artículo "The Notitia Intuitiva of Non-Existents According to William Ockham" de Philotheus Böhner. ${ }^{1}$ Este autor y sus adeptos han basado sus argumentos en una premisa: el conocimiento intuitivo es distinto de un acto

${ }^{1} \mathrm{Ph}$. Böhner, "The Notitia Intuitiva of Non-Existents According to William Ockham: With a Critical Study of the Text of Ockham's Reportatio and a Revised Edition of Rep. II.Q.14-15”, pp. 223-275. 
de asentimiento. En este ensayo me propongo mostrar lo contrario y, en consecuencia, que la epistemología ockhamista lleva a conclusiones escépticas. Mi argumento recae en la interpretación que propongo del desarrollo de los procesos cognoscitivos de singulares expuesto en los Quodlibeta Septem.

En la primera sección de este ensayo explico cuáles son los elementos básicos que participan en todo conocimiento de singulares. Esto, a modo de que el lector pueda apreciar el escenario en el cual se desarrollan los procesos cognoscitivos según la teoría epistemológica ockhamista y se familiarice con los actores principales. Tal escenario, cabe señalar, lo constituyen las dos almas que, según Ockham, conforman a todo ser humano: el alma intelectiva y el alma sensitiva.

En la segunda sección, explico el lugar que ocupan las visiones sensitivas, las visiones intelectivas, los actos de asentimiento simple, los actos de asentimiento complejo y los actos de aprehensión en el desarrollo de los procesos cognoscitivos de singulares. En la tercera, concluyo que un conocimiento intuitivo y un conocimiento abstractivo son actos de asentimiento simple.

En la siguiente sección explico en qué consiste el problema teológico contenido en la teoría del conocimiento intuitivo y abstractivo de Guillermo de Ockham. También expongo cómo tal problema se convirtió en un problema epistemológico.

Con base en lo expuesto en la segunda y la tercera secciones, muestro en la quinta que el problema escéptico no fue resuelto por el Venerabilis Inceptor. ${ }^{2}$

En la última sección expongo otra vía por medio de la cual es posible probar que la epistemología ockhamista tiene conclusiones escépticas. Esta vía apela a la concesión hecha por Ockham de que nuestros sentidos pueden engañarnos.

\section{Los deseos, las habilidades y los hábitos}

en el anima sensitiva $y$ en el anima intellectiva

Todas las habilidades y todos los deseos de cualquier ser humano tienen como sujeto al alma sensitiva o al alma intelectiva. La existencia de estas dos almas posibilita, por una parte, que todo ser humano tenga

${ }^{2}$ Venerabilis Inceptor fue el sobrenombre que se dio a Guillermo de Ockham cuando recibió el grado de "bachiller formado" o "licenciado" en una ceremonia llamada Inceptio. Tal sobrenombre se debió a que, no obstante la ceremonia, Guillermo de Ockham no obtuvo el derecho de cátedra en Oxford. Esto, según Christophe Grellard, debido a la falta de plazas. 
impulsos desiderativos contradictorios entre sí respecto de una misma cosa. Por otra, que todo ser humano posea habilidades de percepción o sensitivas, de imaginación, de entendimiento, etcétera. En este ensayo no trataré sobre los deseos y las pasiones que impulsan a cualquier ser humano, según Ockham, a actuar de alguna u otra forma, sino exclusivamente sobre las habilidades que están directamente involucradas en todo proceso cognoscitivo de singulares.

Así pues, por una parte, una persona es capaz de imaginar y de percibir o sentir objetos externos debido a la existencia del alma sensitiva y de su habilidad de aprehensión. Los actos de imaginación son causados por los sentidos internos, mientras que los actos de percepción o sensaciones son causados por algún objeto externo singular a través de uno o varios de nuestros cinco sentidos. Por otra parte, una persona es capaz —en primera instancia - de tener actos de entendimiento debido a la existencia del alma intelectiva y su habilidad de entendimiento. Según Ockham, $(\alpha)$ si el alma sensitiva y el alma intelectiva fueran lo mismo, cualquier acto de percepción o sensación sería un acto de entendimiento. $^{3}$

El alma sensitiva y el alma intelectiva no sólo son los sujetos de diversos actos, sino también de distintos tipos de hábitos. Cualquier acto del alma sensitiva o del alma intelectiva puede causar un hábito. Un hábito, en un sentido amplio según Ockham, existe en el cuerpo, o bien, es parte de las habilidades aprehensivas, y es causado por un acto y por alguna otra cosa. En un sentido estricto, un hábito es causado sólo por un acto y existe exclusivamente en la voluntad. ${ }^{4}$ De acuerdo con Ockham, un hábito es necesario para: 1) causar actos sin dificultad; 2) causar actos más intensos; 3 ) causar actos similares a aquellos que fueron responsables de la generación del hábito en cuestión, es decir, para causar actos, y 4) inclinar a la voluntad. ${ }^{5}$

En aquellos procesos cognoscitivos que tienen como objeto singulares que están ausentes o han sido destruidos, están involucrados los hábitos intelectivos. Tales hábitos causan actos similares a aquellos que

3 "La premisa menor [i.e. el alma sensitiva y el alma intelectiva son distintas] es probada por el hecho de que si no fuera verdadera, luego cualquiera de las aprehensiones del alma sensitiva serían un acto de entendimiento, en tanto cada una existiría subjetivamente en el alma intelectiva. Similarmente, si las sensaciones existen subjetivamente en el alma intelectiva, luego un alma separada sería capaz de sentir" (Quod. II.10, 47-53; las cursivas son mías).

${ }^{4}$ Quod. II.18, 11-29.

${ }^{5}$ Quod. III.20, 65-73. 
fueron responsables de haberlos causado en un primer momento. ${ }^{6}$ Todo hábito en el alma intelectiva es una cualidad absoluta y un conocimiento, porque todo conocimiento en cuanto hábito intelectivo es algo que se suma o añade al alma intelectiva. ${ }^{7}$

En resumen, según Ockham, el alma sensitiva —desde un punto de vista epistemológico - es el sujeto de la habilidad de aprehensión: la habilidad que posibilita los actos de percepción o sensaciones por medio de nuestros sentidos. El alma intelectiva, en cambio, es el sujeto de la habilidad intelectiva que posibilita - en primera instancia- los actos de entendimiento. Si el alma sensitiva y el alma intelectiva fueran lo mismo, reitero, cualquier acto de percepción o sensación sería un acto de entendimiento.

\section{El proceso cognoscitivo de singulares}

\subsection{Las visiones sensitivas y las visiones intelectivas}

La primera fase de todo proceso cognoscitivo es un acto de percepción o sensación causado por un objeto singular externo. Un acto de percepción podría realizarse por medio del sentido de la vista y así, un acto de este tipo puede interpretarse como una visión sensitiva. ${ }^{8}$

Toda visión sensitiva debe causar una visión intelectiva. Según nuestra interpretación, tal relación causal constituye la segunda fase de todo proceso cognoscitivo de objetos singulares externos y es necesaria porque ningún proceso cognoscitivo podría desarrollarse en el alma sensitiva, sino sólo en el alma intelectiva. Ockham prueba la diferencia entre las visiones sensitivas y las visiones intelectivas por medio de tres vías.

1. Por medio de la experiencia: un niño puede tener visiones sensitivas, pero no visiones intelectivas.

${ }^{6}$ De acuerdo con los cuatro motivos por los cuales es necesario un hábito, un hábito intelectivo es necesario por la razón (3).

${ }^{7}$ Quod. III.20, 47-56.

${ }^{8}$ Apelo al sentido común para entender una visión sensitiva como un acto de percepción o sensación. Apelo, además, a la historia misma del término. Para Peter Aureolo, una visión es una percepción: "información de los sentidos"; así también para Duns Escoto. Ockham aprendió la teoría epistemológica escotista, sobre todo la distinción entre conocimiento intuitivo y conocimiento abstractivo que, como veremos, está directamente vinculada con el concepto latino visio. Apelo, además, a un fragmento que citaré más adelante dentro del cuerpo del texto. Para más información sobre la historicidad del concepto visio, véase: C.H. Tachau, Vision and Certitude in the Age of Ockham: Optics, Epistemology and the Foundations of Semantics, 1250-1345. 
2. Por medio de la razón: un alma separada o un ángel puede tener una visión intelectiva, pero no una visión sensitiva. ${ }^{9}$

3. Si uno sostiene que el alma sensitiva es la misma forma que el alma intelectiva —dice Ockham-, luego no debería afirmar que una visión sensitiva es recibida en el alma intelectiva, sino debería afirmar, en cambio, que es recibida en el cuerpo o en algún poder en el cuerpo que está derivado del alma. Si fuera recibida en el alma intelectiva, luego un alma separada sería capaz, al menos por el poder de Dios, de poseer dentro de ella misma toda sensación de los cinco sentidos, lo cual no se ve verdadero. Si fuera verdadero, luego un ángel siempre estaría careciendo de alguna perfección natural. ${ }^{10}$

La tercera prueba muestra textualmente que es lícito entender que una visión sensitiva es un acto de percepción o sensación. Según tal prueba, una sensación o percepción es equivalente a una visión sensitiva que, según lo dicho antes, sólo puede tener como sujeto al alma sensitiva, pues los sentidos corresponden exclusivamente a ella. La tercera prueba, además, es la misma que Ockham utiliza para distinguir un acto de percepción de un acto de entendimiento $(=\alpha)$. Luego, según nuestra interpretación, es posible afirmar, por analogía entre la tercera prueba y $(\alpha)$, que si una visión sensitiva es un acto de percepción o sensación, una visión intelectiva es un acto de entendimiento.

Según ambas pruebas —el argumento $(\alpha)$ y el argumento que constituye la vía número (3) con la cual Ockham distingue dos tipos de visiones distintas - una visión sensitiva y una visión intelectiva difieren porque la primera sólo puede tener como sujeto al alma sensitiva, mientras que la segunda sólo puede tener como sujeto al alma intelectiva. En este sentido, un ángel o un alma separada no pueden tener una visión sensitiva porque carecen de sentidos externos por cuanto carecen de cuerpo. No obstante, según Ockham, un ángel o un alma separada pueden tener una visión intelectiva y, de este modo, la experiencia de una cosa singular externa. Entonces, un ser humano y un ángel pueden conocer una misma cosa por medio de una visión sensitiva o de una visión intelectiva, respectivamente. Tales visiones o actos - acto de percepción y acto de entendimiento- se distinguen porque pertenecen a diferentes sujetos, no por su contenido. Ambas visiones son similares porque representan cosas externas en dos sujetos diferentes. Una representa singulares en el alma sensitiva; la otra, en el alma intelectiva.

\footnotetext{
${ }^{9}$ Quod. I.15, 36-41.

${ }^{10}$ Quod. I.15, 17-26; las cursivas son mías.
}

Diánoia, vol. LIV, no. 63 (noviembre 2009). 
Según Ockham, una visión sensitiva - junto con el objeto singular externo- causará una visión intelectiva siempre y cuando exista un cuerpo. Tal relación causal es necesaria porque todo acto cognoscitivo, reitero, debe tener como sujeto al alma intelectiva; es imposible un proceso cognoscitivo en el alma sensitiva. Una visión sensitiva debe causar una visión intelectiva para que pueda desarrollarse un proceso cognoscitivo y, si acaso no existieran los sentidos externos - como en un ángel o un alma separada-, un acto de entendimiento o visión intelectiva tendría que ser causado por la sola cosa singular externa. ${ }^{11}$

Pues bien, si una visión intelectiva es una representación de un objeto singular externo causada por una visión sensitiva —según nuestra interpretación-, entonces una visión intelectiva debe ser entendida como un signo: ${ }^{12}$ como un signo simple o un acto de entendimiento. En efecto, en los Quodlibeta, ${ }^{13}$ Ockham expone de un modo acabado su teoría de los actos de entendimiento como signos. Todo acto de entendimiento es un signo simple que representa un singular: un signo que nos hace pensar (= significa) algo distinto del mero acto de entendimiento. ${ }^{14}$ Según lo expuesto en los Quodlibeta, un acto de entendimiento puede formar parte de una proposición. ${ }^{15}$

Toda proposición está formada por signos o conceptos y puede formularse debido a la habilidad de aprehensión del alma intelectiva. En efecto, en el alma intelectiva existen otros tipos de actos además de los de entendimiento; por ejemplo, los actos de formular una proposición, los actos de un primer tipo de asentimiento y los actos de un segundo tipo de asentimiento. Tales actos son también posibles por la habilidad de aprehensión del alma intelectiva y están involucrados en todo proceso cognoscitivo de singulares.

${ }^{11}$ Quod. I.15, 27-31.

${ }^{12}$ Un signo para Ockham es un concepto. En la teoría del conocimiento de madurez del Venerabilis Inceptor cualquier signo — simple o complejo- no es distinto de un acto intelectivo. Un signo o concepto simple es un acto de entendimiento del alma intelectiva. Quod. VI.29, 38-50.

${ }^{13}$ Utilizo como fuente los Quodlibeta Septem porque este libro es conocido como uno de madurez y en él Ockham expuso de un modo acabado su teoría de los signos como actos de entendimiento. Esta teoría, además, aparece en los Quodlibeta Septem ya armonizada con su teoría epistemológica. Por último, es en este libro donde Ockham discute con mayor detenimiento el problema de que Dios puede causar en nosotros un acto de conocimiento.

${ }^{14}$ Para una explicación más amplia de los signos en la teoría epistemológica de Guillermo de Ockham, véase C. Panaccio, Ockham on Concepts.

${ }^{15}$ Quod. IV.35, 115-119. 
Pues bien, Ockham distingue dos tipos de actos de asentimiento: los del primer tipo constituyen conocimientos simples; los del segundo, conocimientos complejos. Veamos en qué consiste esta clasificación.

\subsection{Los actos de asentimiento y los actos de aprehensión}

En el libro III de los Quodlibeta, Ockham distingue ciertos actos de asentimiento o juicio de un primer tipo, llamémoslos (A), de otros actos de asentimiento o juicio de un segundo tipo, llamémoslos (B). La primera distinción dice: a través de (A) una persona puede saber que algo es tal o cual cosa, mientras que a través de (B) una persona puede saber algo en el sentido en que refiere a una cosa. ${ }^{16}$ La segunda distinción señala: mientras un acto (A) tiene objetos singulares externos como objetos, un acto (B) tiene signos complejos o proposiciones como objetos. ${ }^{17} \mathrm{La}$ tercera distinción dice: mientras que un acto $(\mathrm{A})$ no es propiamente un acto de conocimiento - aunque así puede ser entendido-, un acto (B) es propiamente un conocimiento. ${ }^{18}$ Por último, en el libro V de los Quodlibeta Ockham explicita: mediante un acto (A) el intelecto asiente a la existencia de algo (duplex est assensus: unus quo intellectus assentit aliquid esse vel aliquid esse... ), mientras que mediante un acto (B) esto es imposible. ${ }^{19}$

Por medio de un acto (A) una persona puede saber, por ejemplo, que una roca no es un mono; en este sentido, los objetos de un acto (A) son objetos singulares externos. Sin embargo, señala Ockham, es imposible tener en el intelecto singulares externos como objetos ${ }^{20}$ - una roca o un mono-. Entonces, todo conocimiento tiene signos como objetos. En el caso de (A), signos simples de objetos singulares externos. Un signo simple de un singular externo, según lo expuesto en el apartado anterior, es una visión intelectiva causada por una visión sensitiva: un acto de entendimiento causado por un acto de percepción que ha sido causado, a su vez, por un objeto singular externo a través de los sentidos externos del alma sensitiva.

Pues bien, un acto (A) no puede tener como objeto un signo complejo debido a que, según Ockham: 1) tal acto puede existir mediante la formulación de un signo complejo, pero sin la aprehensión de un signo

${ }^{16}$ Quod. III.8, 13-18.

${ }^{17}$ Quod. V.6, 16-18.

${ }^{18}$ Quod. III.8, 27-29. Además, Quod. III.8, 31-34. Un signo complejo es el objeto de un acto de asentimiento del segundo tipo por el cual algo es sabido.

${ }^{19}$ Quod. III.8, 11-18. Para un acto (B) es imposible asentir a la existencia de algo debido a que un acto tal siempre tiene como objetos proposiciones y no cosas.

${ }^{20}$ Quod. III.12, 19-27. 
complejo, y 2) debido a que una persona ordinaria puede realizar un acto (A) sin ser consciente de que está asintiendo a una proposición. ${ }^{21}$ $\mathrm{Si}$ (A) puede existir mediante un acto de formular una proposición, luego un signo complejo no es su objeto, sino su medio. El verdadero objeto de un acto (A) es siempre un signo de un objeto singular externo: un signo simple y, específicamente, un signo simple aprehendido. El objeto de un acto (B), en cambio, es un signo complejo aprehendido.

Todo acto (A) es un conocimiento simple (notitia incomplexa) porque su objeto es siempre un signo simple; todo acto (B) es un conocimiento complejo (notitia complexa) porque su objeto es siempre un signo complejo, es decir, una proposición.

\subsection{Los actos de aprehensión}

No sólo los actos de asentimiento son posibles por la habilidad de aprehensión del alma intelectiva, sino también los meros actos de aprehensión. Según Ockham, hay dos tipos de actos de aprehensión. El primero - llamémoslo (a) - es definido claramente: un acto de formular una proposición; el segundo - llamémoslo (b) —, en cambio, es definido de un modo oscuro: el conocimiento de una proposición (cognitio ipsius complexi iam formati). ${ }^{22}$ Ockham explica ambos actos cuando los distingue de los actos de asentimiento (A) y (B). Según nuestra interpretación, las pruebas (3), (4) y (5) indican que un acto (b) es un hábito de una proposición formulada.

Ockham distingue (A) y (B) de (a) y (b) del modo siguiente:

1. Los actos (A) y (B) son diferentes de (a) porque una proposición formulada es equivalente a una conclusión de una demostración y, en este sentido, según Ockham, a un acto dubitativo. (a) es un acto de creencia (actus credendi) o acto dubitativo; mientras que todo acto de asentimiento tal como cualquier acto (A) o (B) es un acto de conocimiento. $^{23}$

${ }^{21}$ Quod. III.8, 20-27.

${ }^{22}$ Quod. V.6, 20-23.

${ }^{23}$ Por cuanto un acto (a) — un acto de formular una proposición- es un acto de creencia, y un asentimiento (A) un acto por el cual juzgamos que una proposición es verdadera, un asentimiento (A), según nuestra interpretación, es una creencia verdadera: un acto que otorga un juicio de verdad a una proposición o creencia previamente formulada por (a). Más adelante veremos que esta interpretación armoniza clara y finamente con el proceso que se desarrolla en cada acto reflexivo.

Diánoia, vol. LIV, no. 63 (noviembre 2009). 
2. Un acto (A) y un acto (B) son distintos de (a) porque un acto de creencia o acto de formular una proposición está compuesto de partes o de términos, mientras que no así todo acto de asentimiento. ${ }^{24}$

3. Un acto (B) es diferente de (b), porque todo conocimiento de una proposición formulada $(=b)$ es causa de (B). La prueba es que si alguien aprehende una proposición y no asiente a ella en un primer momento a través de un acto (B), y luego por un término medio la misma persona asiente a la misma proposición, ello será porque el intelecto tendría un hábito de (a); no porque el intelecto tuviera un hábito de (B), puesto que un hábito no puede existir después de un solo acto de asentimiento. En conclusión, un acto (B) y otro (b) difieren debido a que un hábito de (a) - un conocimiento de una proposición formulada - puede causar (B). Esta conclusión coincide con las pruebas restantes para distinguir los actos (A) y (B) de (b).

4. Ambos actos de asentimiento son distintos de (b) porque cualquier persona puede tener un hábito de una proposición formulada que no ha sido asentida o disentida, es decir, un conocimiento en cuanto hábito de un acto (a) neutro.

5. Finalmente, todo acto de asentimiento difiere de (b) porque cualquier persona puede asentir consecutivamente a (b) y después disentir respecto de la misma proposición. ${ }^{25}$ Esto sólo es posible si el objeto de asentimiento es un hábito y no un acto.

Los tres últimos puntos de la lista anterior, según lo señalamos antes, indican que un acto (b) es un conocimiento de una proposición en cuanto hábito de una proposición. Tal conclusión está apoyada en un par de afirmaciones más. La primera de ellas fue expuesta en el primer apartado de este ensayo: todo hábito es un conocimiento y una cualidad y, en cuanto tal, existe en las habilidades de aprehensión. La segunda afirmación evoca los objetos de conocimiento de los actos (A) y (B); para explicar esto último debemos hacer una digresión.

Según Ockham, todo acto (A) implica la previa aprehensión de un signo simple; mientras que todo acto (B) implica la previa aprehensión de un signo complejo. ${ }^{26}$ Todo acto de juicio presupone la aprehensión de su propio objeto de conocimiento, el cual en todos los casos es un signo. La diferencia entre los actos (A) y (B) es que (A) requiere

\footnotetext{
${ }^{24}$ Quod. V.6, 26-28, 37-43.

${ }^{25}$ Quod. V.6, 45-47, 59-75.

${ }^{26}$ Quod. IV.16, 40-43.
} 
la aprehensión de un signo simple. ${ }^{27}$ En cambio, un acto (B) requiere la aprehensión de un signo complejo. Si un hábito puede entenderse como parte de las habilidades aprehensivas —según lo señalado en el primer apartado de este ensayo-, entonces, un hábito es un acto de aprehensión. ${ }^{28}$ Cualquier acto de juicio — sea (A) o (B) — implica un hábito previo causado por el mero acto de entendimiento - signo simple- o el acto de formular una proposición —-signo complejo-. Si (b) es un acto de aprehensión de un signo complejo, entonces, (b) es un hábito de formular una proposición; un hábito como el mencionado en la distinción (2) entre un acto (B) y (b).

Ahora bien, ¿por qué todo acto de juicio presupone la aprehensión de su propio objeto de conocimiento? Según Ockham, esto se debe a que en el intelecto es imposible que existan más de dos actos presentes al mismo tiempo. ${ }^{29}$ Veamos cómo se desarrollaría un juicio dentro de un proceso cognoscitivo.

Cuando un acto (A) existe en el intelecto en el presente, un acto (a) debe existir al mismo tiempo, pues (A) presupone un acto de formular una proposición. ${ }^{30}$ Una proposición está formada por distintos signos simples o actos de entendimiento, los cuales no podrían existir como actos si estuvieran presentes un acto (A) y otro acto (a) al mismo tiempo. Los objetos de (A) tendrían que existir, entonces, como hábitos; no como actos. ${ }^{31}$ Es decir, deben ser aprehendidos previamente para que los actos (A) y (a) sean posibles al mismo tiempo. Lo mismo ocurre cuando se realiza un acto de asentimiento del segundo tipo. Cuando un acto (B) existe en el intelecto en el presente, un acto (A) debe existir al

${ }^{27}$ Quod. IV.16, 32-36. El intelecto siempre asiente a signos de cosas singulares.

${ }^{28}$ Los hábitos que un alma sensitiva o un alma intelectiva posee son, en un sentido aristotélico, aquello opuesto a los actos. Los actos son la operación o el ejercicio de los hábitos; un hábito como cualidad es un acto en potencia. Para una mayor documentación sobre el sentido aristotélico de los actos en la epistemología ockhamista, véase C. Panaccio, op. cit., p. 21.

${ }^{29}$ Quod. I.14, 47.

${ }^{30}$ Recuérdese que la formulación de una proposición es el medio por el cual se realiza un acto (A). En este sentido, todo acto (A) presupone una proposición.

${ }^{31}$ Cuando realizamos un acto (A) formulamos, según Ockham, una proposición tal como: “'una piedra no es un mono' es verdadera". Es decir, formulamos nuevamente una proposición —compuesta de signos simples- que debió haber sido formulada previamente por (a) — "una piedra no es un mono"- y añadimos a ella un valor de verdad. Todo acto (B) presupone un acto de formular una proposición con un signo complejo; por ejemplo, "'una piedra no es un mono' es verdadera". En este sentido, un acto (B) tiene un signo complejo como objeto y presupone (A). 
mismo tiempo en él. Luego, un acto (a) —así como los actos de entendimiento que lo componen- no podría existir como tal en el intelecto al mismo tiempo, sino como hábito: como signo complejo aprehendido $(=\mathrm{b})$.

En suma, según mi interpretación, un acto de aprehensión del alma intelectiva puede ser entendido como (i) un acto de formular una proposición: un acto de creencia $(=\mathrm{a})$; (ii) un hábito de un signo complejo formulado $(=\mathrm{b})$, y (iii) un hábito de un signo simple, es decir, de un acto de entendimiento. Los casos (i) a (iii) son todos actos de aprehensión porque son posibles dada la habilidad de aprehensión del alma intelectiva.

Así las cosas, tenemos que dentro de un proceso cognoscitivo de un objeto singular externo, un acto (a) sigue a un acto de entendimiento, pues toda proposición está formada por signos simples. El acto de formular una proposición es la tercera fase dentro de todo proceso cognoscitivo de un objeto singular externo. La cuarta fase, en cambio, es un acto de asentimiento del primer tipo $(=\mathrm{A})$ : un acto que siempre presupone la formulación de una proposición. Cualquier acto (A) podría ser seguido por (B). Si éste es el caso, (B) constituiría la última fase de un conocimiento. Un acto (B), según Ockham, es propiamente un conocimiento en cuanto que tiene signos complejos o proposiciones como objetos.

Todo proceso cognoscitivo de singulares se desarrolla, según nuestra interpretación, como se muestra en el diagrama de la p. 101.

\section{El conocimiento intuitivo y el conocimiento abstractivo}

En el libro I de los Quodlibeta Ockham dice: "Una visión sensitiva no es suficiente para causar un asentimiento de una proposición contingente; en cambio, es suficiente para causar un acto en el apetito sensitivo."32 Una visión sensitiva no es suficiente para causar un asentimiento de una proposición contingente porque una visión tal nunca existe en el alma intelectiva -único sujeto en el que puede desarrollarse un proceso cognoscitivo, según lo hemos reiterado-. Una visión sensitiva debe causar una visión intelectiva que posteriormente podrá causar un asentimiento $(=\mathrm{A})$ por medio del acto de formular una proposición $(=\mathrm{a})$. Pero ¿qué es una tal proposición contingente (propositio contingente)? Una proposición contingente es aquella que refiere a la existencia presente

${ }^{32}$ Quod. I.15, 27-29; las cursivas son mías.

Diánoia, vol. LIV, no. 63 (noviembre 2009). 
de una cosa; por ejemplo: "esta blancura existe". ${ }^{33}$ De acuerdo con lo especificado en la cita anterior y con lo establecido antes, una proposición como "esta blancura existe" presupone una visión sensitiva y una visión intelectiva; es formulada por un acto (a) y puede estar seguida por un acto de asentimiento (A). Todo acto de asentimiento (A) tiene un signo de un singular como objeto - por ejemplo, una blancura o una roca-; se realiza mediante un acto (a) - por ejemplo, "esta blancura existe"- y puede referir a la existencia de algo (duplex est assensus: unus quo intellectus assentit aliquid esse...).

Ahora bien, según Ockham, todo conocimiento intuitivo (i) presupone la formulación de una proposición que refiere a la existencia presente de un singular; (ii) es un conocimiento simple; (iii) es un conocimiento propio con respecto a una cosa singular, y (iv) es primero respecto de un conocimiento universal. ${ }^{34}$ De acuerdo con (i) y (ii), un conocimiento intuitivo es claramente un acto (A): un conocimiento simple que presupone la formulación de una proposicion $(=\mathrm{a})$, la cual puede referir a la existencia presente de algo (duplex est assensus: unus quo intellectus assentit aliquid esse...).

Pero la identidad entre un acto (A) y un conocimiento intuitivo no es obvia si consideramos (iii) y (iv). Esto es así porque, según nuestra interpretación, no todo acto (A) es un conocimiento intuitivo, pero todo conocimiento intuitivo es un acto (A). En efecto, por medio de un acto (A) podemos saber que algo existe actualmente porque tal conocimiento ha sido causado por una cosa singular; es decir, porque es propio con relación a una cosa singular, tal como todo conocimiento intuitivo. Además, si un acto (A) es causado por una cosa singular, necesariamente debe ser anterior a un conocimiento universal, pues todo conocimiento de este tipo, de acuerdo con el nominalismo ockhamista, es producido por nuestra habilidad intelectiva de hallar similitudes entre diversos singulares previamente conocidos. Un acto (A) no es necesa-

${ }^{33}$ Según Ockham, una persona asiente a una proposición contingente o a una proposición primaria contingente sólo por un conocimiento intuitivo. Si por un conocimiento intuitivo siempre asentimos a la existencia de un singular externo, entonces, una proposición primaria contingente y una proposición contingente refieren a la existencia de un singular externo. Ambos tipos de proposiciones no difieren por su contenido. Según nuestra interpretación, difieren porque una proposición primaria contingente es una que nunca ha sido formulada por el intelecto previamente, mientras que una proposición contingente es una proposición que ya antes fue formulada.

34 “[A] firmo que un conocimiento que es simple, propio para el singular, y primero por el tipo de primacía en cuestión [i.e., respecto de un conocimiento universal], es un conocimiento intuitivo" (Quod. I.13, 38-39). 


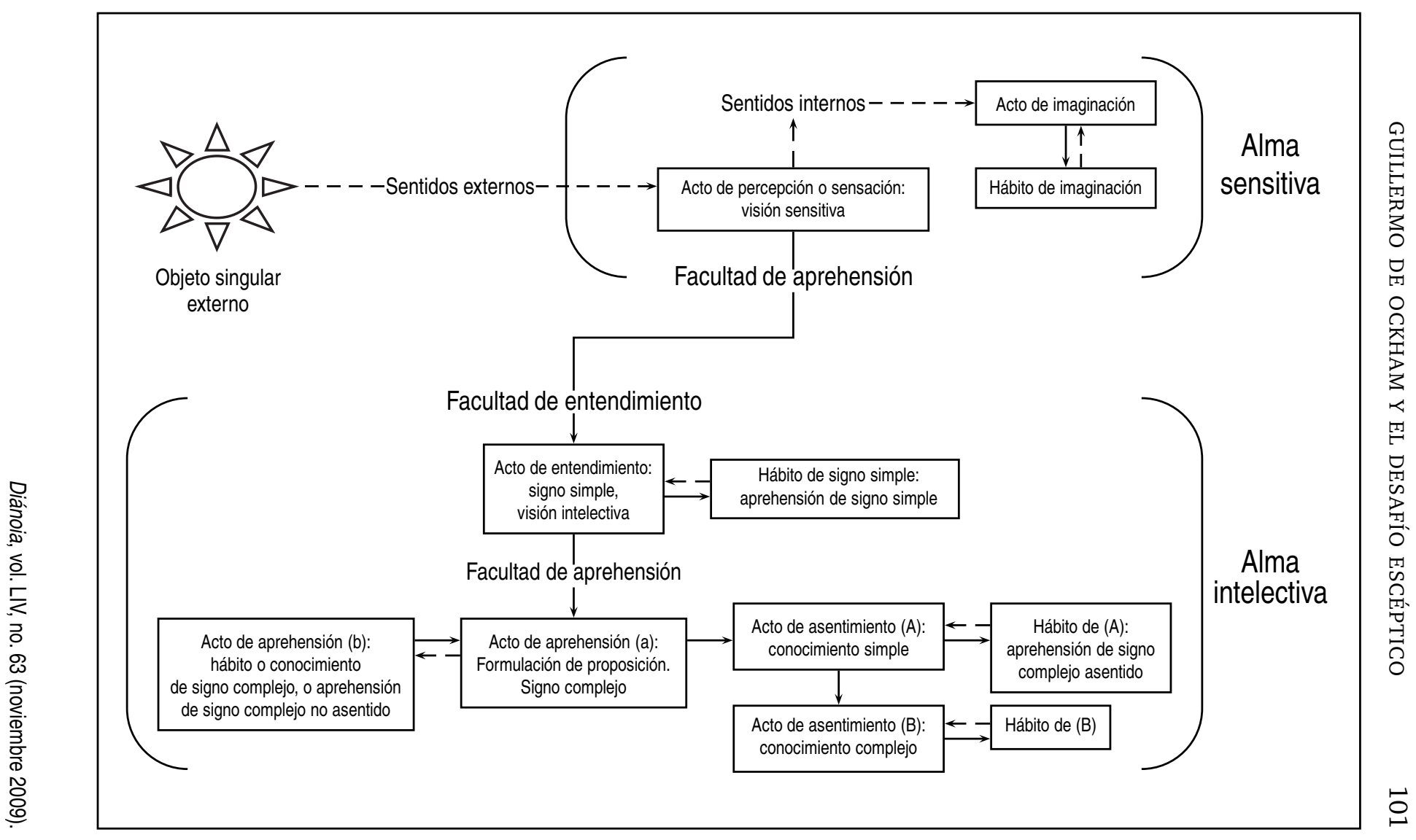


riamente propio ni anterior con respecto a un conocimiento universal; aunque todo conocimiento intuitivo sí lo es.

Pero hay más pruebas para apoyar nuestra interpretación de que un conocimiento intuitivo es una forma específica de un acto (A): un acto de asentimiento respecto de la existencia actual de una cosa. En el libro V de los Quodlibeta, Ockham caracteriza el conocimiento intuitivo en relación con el conocimiento abstractivo en un párrafo central que en latín dice:

$(\beta)$ Et dico ad praesens quod dupliciter different: uno modo per hoc quod per notitiam intuitivam assentitur primo contingenti, et per abstractivam non; alio modo per hoc quod per notitiam intuitivam non tantum iudico rem esse quando est, sed etiam non esse quando non est; per abstractivam neutro modo iudico. (Quod. V.5, 14-19; las cursivas son mías.)

La tradición anglosajona ha traducido al inglés el párrafo anterior del modo siguiente:

$(\gamma)$ I claim that they differ in two ways: in one way, by reason of the fact that through an intuitive, but not through an abstractive cognition, one assents to a primary contingent proposition; in a second way, by reason of the fact that through an intuitive cognition I judge not only that a thing exists, when it exists, but also that it does not exist, when it does not exist; I do not judge in either of these ways through an abstractive cognition. (Las cursivas son mías.)

En $(\gamma)$ se traduce la preposición "per" por "through": "a través de" en español; sin embargo, en latín —así como en español- las preposiciones "per" y "por" no sólo significan "a través de": "in one side and out another side of; in the midst of; by way of", según The American Heritage Dictionary. ${ }^{35}$ En latín tardío ${ }^{36}$ - y por herencia, en el españolcuando se utiliza "per" en las oraciones pasivas tal preposición indica el agente pasivo: el agente que realiza la acción de manera pasiva. ${ }^{37} \mathrm{En}$ $(\beta)$, la oración "per notitiam intuitivam assentitur primo contingenti" está en voz pasiva, tal como lo indica el verbo "assentitur" que tiene la desinencia propia de tal voz para la tercera persona en tiempo presente (-itur). El agente pasivo de la misma oración es "notitiam intuitivam".

${ }^{35}$ The American Heritage Dictionary, p. 852.

${ }^{36}$ J.M. Baños, "Análisis funcional de los sintagmas preposicionales: Per + acusativo en latín clásico", pp. 461-478.

${ }^{37}$ En español, esto lo expresa el Diccionario de la Real Academia de la Lengua Española: "preposición con que se indica la persona agente en las oraciones en pasiva" (Diccionario de la Real Academia de la Lengua Española, pp. 1086-1087).

Diánoia, vol. LIV, no. 63 (noviembre 2009). 
Entonces, "notitiam intuitivam" es el agente que realiza la acción de asentir. No así, tal como lo expresa la traducción inglesa, el lugar por donde, o el medio por el cual se asiente a algo.

Pero en $(\beta)$ también hay una oración activa: "per notitiam intuitivam non tantum iudico rem esse quando est, sed etiam non esse quando non est". El verbo "iudico" está en voz activa, primera persona, singular, en tiempo presente; sin embargo, es un verbo de pensamiento y no de movimiento, lo cual, siguiendo a J.M. Baños, nos permite interpretar que el sustantivo regido por la preposición "per" —el complemento circunstancial- indica ubicación y no lugar por donde ${ }^{38}$ debido a la herencia del latín clásico. Un conocimiento intuitivo, entonces, no es el medio por el cual asentimos, sino el asentimiento mismo respecto de una proposición contingente. Un conocimiento intuitivo es un asentimiento por el cual sabemos que una cosa existe.

De acuerdo con lo anterior, el párrafo $(\beta)$ debe traducirse del modo siguiente:

$(\delta)$ ahora afirmo que difieren en dos sentidos: uno, por razón de que por un conocimiento intuitivo, pero no por un conocimiento abstractivo, se asiente a una proposición primaria contingente; en un segundo sentido, por razón de que por un conocimiento intuitivo yo juzgo no sólo que una cosa existe, cuando existe, sino también que no existe cuando no existe; y no juzgo en cualquiera de estos sentidos por un conocimiento abstractivo. (Las cursivas son mías.)

Traducido así, es evidente que un conocimiento intuitivo es un acto de asentimiento (A): un acto de asentimiento es un acto de juicio.

Ahora bien, un conocimiento intuitivo y un conocimiento abstractivo son actos de distinta especie; sin embargo, ambos actos tienen singulares como objetos. ${ }^{39}$ Luego, según nuestra interpretación, ambos actos

38 "[P]er + accus., en el campo de las relaciones espaciales, puede expresar no sólo (como se afirma habitualmente) Lugar por donde sino también Ubicación [. . .]; el criterio para determinar cuándo esta preposición es marca de una u otra función radica en la naturaleza de la acción (dinámica o estática) expresada por el predicado verbal" (J.M. Baños, op. cit., p. 478). Agustín Mateos Muñoz también asevera que la preposición per + accus. puede interpretarse como Ubicación o Lugar, y no necesariamente como Lugar por donde o Medio de (A. Mateos, Gramática latina, p. 169).

39 “[P] numéricamente el mismo objeto. Esto es evidente en el caso de un conocimiento intuitivo y un conocimiento abstractivo" (Quod. II.18, 69-71). Recuérdese que todo objeto singular es un signo simple en el alma intelectiva. 
son dos formas distintas en las que un acto de asentimiento (A) se realiza. Esto explica por qué todo conocimiento intuitivo y abstractivo es un acto (A), pero no todo acto (A) un conocimiento intuitivo, o bien, un conocimiento abstractivo exclusivamente. Las diferencias entre ambos tipos de conocimiento son, según Ockham: 1) un conocimiento abstractivo presupone un conocimiento intuitivo respecto del mismo objeto, pero no viceversa; ${ }^{40}$ 2) por un conocimiento abstractivo no podemos asentir a una proposición primaria contingente; ${ }^{41} 3$ ) un conocimiento abstractivo no es suficiente para causar un conocimiento complejo evidente, ${ }^{42}$ y 4) por un conocimiento abstractivo no podemos asentir a la existencia presente de una cosa. ${ }^{43}$

Un conocimiento abstractivo presupone que sabemos que algo existe. En este sentido, presupone un conocimiento intuitivo. Por un conocimiento abstractivo no podemos juzgar que una cosa existe, porque un conocimiento tal es causado por un hábito que alguna vez fue causado por un acto de entendimiento que representó a una cosa singular. Un conocimiento abstractivo nunca podrá ser causado sólo por un objeto singular externo presente. Por un conocimiento abstractivo no podemos asentir a una proposición contingente, porque no presupone una proposición que refiera a la existencia presente de una cosa, sino una proposición que refiere a una cosa singular, tal como todo acto de asentimiento (A). Un conocimiento abstractivo es un conocimiento simple porque sus objetos son singulares causados por hábitos y, en este sentido - tal como todo conocimiento intuitivo-, todo conocimiento abstractivo es un acto (A); aunque no todo acto (A) es un conocimiento abstractivo: un acto (A) puede ser también un conocimiento intuitivo. Ambos tipos de conocimiento son causados por singulares, pero difieren debido a la naturaleza del singular que los causa de manera primordial. Un conocimiento intuitivo es un acto (A) de un singular que existe realmente. Un conocimiento abstractivo es un acto (A) de un hábito de un singular que existe en el alma intelectiva.

Según Ockham, si un conocimiento abstractivo fuera causado solamente por un hábito, sería un conocimiento simple pero común, es decir, no propio: causado por una cosa singular externa no específica. Un conocimiento abstractivo con tales características ocurriría cuando un conocimiento intuitivo fuera imposible; por ejemplo, cuando la cosa no existiera o también, según Ockham, cuando viéramos una cosa

${ }^{40}$ Quod. I.13, 38-41.

${ }^{41}$ Véase $(\delta)$, también: Quod. V.5, 7-10.

${ }^{42}$ Quod. I.15, 10-14.

${ }^{43}$ Quod. I.13, 38-41.

Diánoia, vol. LIV, no. 63 (noviembre 2009). 
que se acerca a la distancia y respecto de la cual no pudiéramos afirmar que es una persona o un animal, sino simplemente: “'aquella cosa es un ser' es verdadera". Un conocimiento tal no sería propio porque no sería causado por un singular específico, sino por un singular que podría ser cualquier otro. ${ }^{44}$ Este caso sería igual al acto de asentir a una proposición tal como "una roca no es un burro" si la roca y el burro no existieran en el presente.

Así como un conocimiento abstractivo puede ser simple pero común, según Ockham, un conocimiento tal también puede ser propio para un singular pero compuesto de signos simples. Es el caso, por ejemplo, del recuerdo de que alguien a quien vemos es Sócrates. Un conocimiento tal sería propio porque estaría causado por una cosa singular específica, pero sería complejo debido a que podríamos recordar que lo que vemos es Sócrates sólo porque tendríamos en la memoria que él tiene tal o cual figura, color y estatura, porque podríamos recurrir a todos estos conceptos para reconocer a Sócrates. ${ }^{45}$

Pero no hemos explicado otra de las características del conocimiento abstractivo. ¿Por qué una proposición contingente no puede ser conocida evidentemente por un conocimiento abstractivo? Cualquier acto (A) puede causar un conocimiento complejo, es decir, un acto (B). Si (A) presupusiera una proposición contingente y el objeto al que refiriera estuviera realmente presente, el acto (A) sería un conocimiento intuitivo y un conocimiento evidente, y así podría causar un conocimiento complejo evidente. Todo conocimiento evidente de una proposición contingente implica que la cosa singular existe y está presente. ${ }^{46} \mathrm{Si}$ el singular no está presente y se realiza un acto (A), este acto será un conocimiento abstractivo, el cual no podrá causar un conocimiento complejo evidente de una proposición contingente, porque habría sido causado por un hábito. En los Quodlibeta Septem, según nuestra interpretación, un conocimiento intuitivo o un conocimiento abstractivo son los actos penúltimos que se realizan en un proceso cognoscitivo. El último acto de un proceso cognoscitivo es un acto (B) que es propiamente un conocimiento, pues, reitero, un acto (B) siempre tiene como objetos signos complejos, es decir, proposiciones.

${ }^{44}$ Quod. I.13, 46-56.

${ }^{45}$ Quod. I.13, 122-133.

46 "[E]l conocimiento evidente implica que las cosas son en realidad como ellas son afirmadas por la proposición por la cual el asentimiento es dado" (Quod. V.5, 64-65). 
4. El desafío escéptico y teológico a la epistemología ockhamista

En el libro V de los Quodlibeta, Ockham explica varias objeciones en contra de su teoría del conocimiento; en particular, en contra de las nociones de conocimiento intuitivo y conocimiento abstractivo. Tales objeciones están basadas en un problema teológico: $(\zeta)$ si por un conocimiento intuitivo sabemos que algo existe cuando existe y está presente, y por un conocimiento abstractivo no podemos saber que algo existe porque tal conocimiento abstrae de la existencia actual, entonces, Dios no sería capaz de causar un conocimiento de algo que no está presente, haciéndolo aparecer como si estuviera presente. ${ }^{47}$ Según el objetor, la conclusión anterior es imposible porque Dios es capaz de hacer cualquier cosa que él deseara.

Aquí no explicaré en qué consisten cada una de las objeciones que se derivan de $(\zeta)$, sino sólo las respuestas que Ockham formuló. Cada respuesta explica cada uno de los medios por los cuales Dios podría causar un conocimiento de una cosa que no está presente haciéndola aparecer como si lo estuviera.

En primer lugar, Ockham concede que Dios es capaz de causar un conocimiento por el cual una cosa que está ausente aparezca como presente; sin embargo, un conocimiento causado por Dios no sería un conocimiento intuitivo, sino un conocimiento abstractivo. ${ }^{48}$ Un conocimiento tal, en principio, no podría ser evidente ${ }^{49}$ y no sería natural. En segundo lugar, Ockham explica que Dios no sólo podría causar un conocimiento abstractivo, sino también una visión sensitiva, haciendo que ésta permanezca de manera no natural en el alma sensitiva. ${ }^{50} \mathrm{Sin}$ embargo, esta visión no podría causar una visión intelectiva y, finalmente, un conocimiento intuitivo, porque el objeto singular externo -aquel elemento necesario para que pueda desarrollarse un proceso cognoscitivo- no existiría realmente. Puesto que un conocimiento intuitivo no podría realizarse a causa de una visión sensitiva falsa, no podríamos tener un conocimiento evidente. ${ }^{51}$

Debido a que los conocimientos causados por Dios no podrían ser evidentes, el objetor pregunta: ¿Dios no es capaz de causar un conocimiento evidente? Ockham responde: Dios no es capaz de causar

${ }^{47}$ Quod. V.5, 61-64.

${ }^{48}$ Quod. V.5, 72-76.

${ }^{49}$ Quod. V.5, 65-71.

${ }^{50}$ Quod. V.5, 52-56.

51 "Dios podría no ser capaz de producir un asentimiento evidente de la proposición contingente 'esta blancura existe' cuando la blancura no exista" (Quod. V.5, 58-59).

Diánoia, vol. LIV, no. 63 (noviembre 2009). 
un conocimiento evidente cuando el objeto no existe realmente en el presente; sin embargo, Dios es capaz de causar un conocimiento del mismo tipo que un conocimiento evidente. ${ }^{52}$ La respuesta no resulta satisfactoria al objetor y, por esta razón, refuta: Dios es capaz de producir un objeto singular externo efímero que podría ser conocido por el intelecto. ${ }^{53}$ Ante esta posibilidad, Ockham dice: el conocimiento de una cosa efímera producida por Dios no sería realmente evidente porque tal cosa no habría sido producida naturalmente ${ }^{54}$ y sería irreal. No obstante, una cosa efímera producida por Dios podría causar una visión sensitiva que, a su vez, causaría una visión intelectiva y, finalmente, un conocimiento que, según los apartados precedentes, no podría ser otro que uno intuitivo: un asentimiento (A) de una proposición contingente. Nada evitaría que un proceso cognoscitivo se siguiera de la existencia no natural, efímera e irreal de una cosa, según concede Ockham; aunque este proceso no desembocaría en un conocimiento evidente.

Ahora bien, en el libro VI de los Quodlibeta, Ockham explica otra objeción similar a $(\zeta)$ pero sutilmente diferente: ¿Dios es capaz de causar un conocimiento intuitivo? El problema es distinto de $(\zeta)$ porque no establece que mediante un conocimiento tal la cosa aparecería aun cuando realmente no existiera. La respuesta ockhamista es: Dios es capaz de causar directamente un conocimiento intuitivo ${ }^{55}$ sin una visión sensitiva o una visión intelectiva. Este conocimiento -así como cualquier conocimiento causado por Dios- no podría ser evidente, pues la cosa a la que referiría como si estuviera presente realmente no existiría ni estaría presente. Además, no podría permanecer naturalmente en el intelecto debido a la ausencia del objeto singular externo, de una visión sensitiva y de una visión intelectiva; sin embargo, tal conocimiento podría permanecer de manera no natural. ${ }^{56}$

Pues bien, de acuerdo con lo hasta aquí explicado, Dios puede engañarnos causando un objeto singular externo efímero. Así también, Dios puede engañarnos causando en nosotros directamente un conocimiento intuitivo o uno abstractivo; es decir, según mi interpretación, causando un acto (A) respecto de una cosa singular aparentemente existente, o un acto (A) respecto de un singular que está ausente pero que aparece

${ }^{52}$ Quod. V.5, 91-100.

${ }^{53}$ Quod. V.5, 105-107.

${ }^{54}$ Quod. V.5, 108-111.

55 " $[\mathrm{P}]$ or el poder de Dios puede haber un conocimiento intuitivo de una cosa que no existe" (Quod. VI.6, 11-13).

${ }^{56}$ Quod. VI.6, 46-51.

Diánoia, vol. LIV, no. 63 (noviembre 2009). 
al intelecto como si estuviera presente. Luego, ni un conocimiento intuitivo ni uno abstractivo es infalible: pueden ser conocimientos falsos. No obstante, si acaso Dios los causara, ninguno de los dos conocimientos sería realmente evidente.

La reiteración ockhamista de que ningún conocimiento causado por Dios puede ser evidente constituye, según nuestra interpretación, el centro de su estrategia para evitar consecuencias escépticas. Ninguna creencia verdadera causada de manera no natural es evidente y, por lo tanto, no está justificada. ¿Cómo podemos justificar que nuestras creencias son verdaderas? ¿Cómo podemos distinguir un conocimiento evidente de otro causado por Dios? La respuesta, según mi interpretación, es la siguiente: a través de actos reflexivos podemos saber con certeza que un conocimiento ha sido causado naturalmente o por Dios. Por un acto reflexivo, según Ockham, podemos saber si una creencia que consideramos verdadera está justificada o no.

5. El conocimiento reflexivo intuitivo y la solución ockhamista al desafío escéptico

Naturalmente, un conocimiento intuitivo directo es un acto que presupone una visión intelectiva, mientras que un conocimiento abstractivo directo es un acto que presupone un hábito. Todo conocimiento directo tiene signos de singulares como objetos, aun si tales singulares están presentes o ausentes. ${ }^{57} \mathrm{Un}$ acto de conocimiento intuitivo reflexivo, llamémoslo (c), presupone un acto intelectivo presente; mientras que un acto de conocimiento abstractivo reflexivo, llamémoslo (d), presupone un acto intelectivo no presente, es decir, un hábito de un acto intelectivo. Un conocimiento intuitivo o un conocimiento abstractivo puede ser el objeto de algún acto reflexivo.

De acuerdo con Ockham, todo acto (d) produce un regreso infinito de conocimientos; no así cualquier acto (c). Esto es verdadero porque un conocimiento abstractivo o un conocimiento intuitivo puede ser conocido por un acto (d) aun cuando no existan actualmente, mientras que un conocimiento intuitivo y un conocimiento abstractivo pueden ser conocidos por un acto (c) si y sólo si están presentes en el intelecto. ${ }^{58}$ Puesto que todo acto (d) causa un regreso infinito de conocimientos y no puede causar ninguna certeza, aquí no explicaré este tipo de actos, sino solamente los actos (c) y el modo en el cual producen - $\mathrm{O}$

${ }^{57}$ Quod. II.12, 18-26.

${ }^{58}$ Quod. II.12, 42-54.

Diánoia, vol. LIV, no. 63 (noviembre 2009). 
tienen que producir - certeza en cuanto a la naturaleza de un conocimiento.

Un acto reflexivo (c) de un conocimiento abstractivo puede producir certeza si un ser humano experimenta un hábito que causó un conocimiento abstractivo. Ockham no explica este caso; sin embargo, infiero que mediante un conocimiento reflexivo (d) de un conocimiento abstractivo uno estaría cierto de tener como verdadera una creencia y de que tal creencia está justificada porque proviene de una demostración bien formulada.

Ahora bien, en cuanto a un conocimiento reflexivo (c) de un conocimiento intuitivo, Ockham dice lo siguiente: si experimentamos una visión intelectiva responsable de un conocimiento intuitivo en el intelecto, un acto reflexivo (c) podrá producir certeza. Un acto (c) de un conocimiento intuitivo, sin embargo, no sólo presupone una visión intelectiva que habría causado un tal conocimiento intuitivo, sino también un hábito como el siguiente: $(\varepsilon)$ "Todo efecto de un mismo tipo tiene causas de un mismo tipo." 59 En otras palabras, para que pueda efectuarse un conocimiento reflexivo (c), debemos tener un hábito $(\varepsilon)$ y una visión de una visión causal de un conocimiento intuitivo para que desarrollemos una demostración como la siguiente:

1. Toda visión es causada por un objeto singular externo;

2. Si sé que yo tengo una visión en mi intelecto, sé que tal visión fue causada por un objeto singular externo;

3. Sé que tengo una visión en mi intelecto;

59 “[E]s por virtud de la visión de la piedra y también por virtud de la visión de la primera visión que yo estoy cierto de que estoy conociendo una roca, y quizá esto es algunas veces por virtud de las dos visiones y también por virtud de alguna proposición que habitualmente es sabida.

Un ejemplo: estoy cierto de que estoy teniendo un conocimiento experimental porque yo veo la visión de la roca. Pero es por el razonamiento del efecto a la causa que estoy cierto de que estoy conociendo una roca, en el sentido de que estoy conociendo un fuego a través de su humo por virtud del hecho de que otras veces he visto el humo causado en la presencia del fuego. En el mismo sentido, por virtud del hecho de que cuando una roca está presente al intelecto, experimento que una visión similar es causada en mí, razono como sigue: estos efectos son de la misma especie; luego, son producidos por causas de la misma especie. [...] La proposición que es sabida habitualmente es como ésta: 'todos los efectos tales de la misma especie tienen causas de la misma especie'. (No estoy haciendo la afirmación general de que todos los efectos de la misma especie tienen causas de la misma especie.)" (Quod. I.14, 77-91). 
4. Sé que tal visión fue causada por un objeto singular externo.

Podemos estar ciertos de que una de nuestras creencias verdaderas o uno de nuestros conocimientos intuitivos está justificado porque podemos ver una visión que causó tal conocimiento y porque sabemos que tal visión fue causada por un singular externo.

Sin embargo, y de acuerdo con lo expuesto anteriormente, tal visión podría haber sido causada por un objeto singular efímero no natural, es decir, un objeto singular causado por Dios. Entonces, la primera premisa del argumento anterior podría ser falsa y, de este modo, podríamos estar ciertos de que nuestro conocimiento intuitivo está justificado y realmente constituye un conocimiento aun cuando no sea así. Podríamos estar ciertos de que nuestro conocimiento es evidente aun cuando el objeto singular externo no exista de modo natural y sea irreal. El intento ockhamista por erradicar el desafío escéptico a través de la noción de conocimiento reflexivo no es terminante. Dios podría engañarnos constantemente sin que nosotros tengamos posibilidad alguna de saberlo.

\section{Es posible que nuestros sentidos nos engañen}

En el libro VI de los Quodlibeta Ockham dice:

Puedes objetar que si alguien ve el sol y después entra a un lugar oscuro, le parecerá que ve el sol en el mismo lugar y con la misma medida. Luego, la visión del sol permanece aun cuando el sol está ausente, por la misma razón esta visión permanecería aunque el sol no existiera.

Replico que no es una visión del sol aquello que permanece; en cambio, lo que permanece es cierta cualidad, viz., la luz impresa en el ojo, y ésa es la cualidad que es vista. Y si el intelecto formula una proposición tal como "la luz es vista en el mismo lugar, etc." y asiente a ella, luego estará siendo engañado por aquella cualidad impresa a la cual ve. (Quod. VI.6, 52-60)

De acuerdo con el párrafo anterior, hay algo que permanece en el cuerpo cuando vemos el sol. Ese algo no es una visión, sino una cualidad en el cuerpo que causaría una visión por la cual nosotros afirmaríamos que vemos el sol del mismo tamaño y en el mismo lugar aun cuando el sol esté ausente. En otras palabras, tal cualidad impresa en el ojo causaría una visión sensitiva que causaría una visión intelectiva que, por último, causaría un asentimiento por medio de un acto de formular una proposición. Un asentimiento tal, de acuerdo con lo expuesto en los apartados anteriores, sería un conocimiento intuitivo. 
Según el pasaje anterior, Ockham concede que podemos ser engañados naturalmente por medio del alma sensitiva y de los sentidos externos. Además, del pasaje anterior se sigue que podríamos estar ciertos de que un conocimiento tal está justificado si tuviéramos un conocimiento intuitivo reflexivo de la visión intelectiva causada por la visión sensitiva, a su vez causada por aquella cualidad impresa en el ojo.

Tal como Pasnau lo asevera, ${ }^{60}$ la solución ockhamista es plausible; no obstante, implica un serio problema: Ockham acepta que podemos ser engañados por nuestros sentidos externos y, por lo tanto, que podemos ser engañados no sólo de un modo no natural - por Dios-, sino también, naturalmente. Además, en los Quodlibeta, Ockham no explica por qué el acto de ver cualquier cosa singular no causaría una cualidad en el ojo tal como la que causaría el acto de ver el sol. Finalmente, reitero, el modo en el cual sería causado un conocimiento por tal cualidad en el ojo permitiría que, por medio de un acto reflexivo, pudiéramos estar ciertos de que nuestra creencia es verdadera y está justificada aun cuando sea falsa.

\section{Conclusiones}

La interpretación que he propuesto aquí de los procesos cognoscitivos de singulares en la teoría epistemológica ockhamista expuesta en los Quodlibeta Septem armoniza con varias ideas.

1) Coincide con la interpretación de $\operatorname{Karger}^{61}$ y Pegis ${ }^{62}$ de que un conocimiento intuitivo y un conocimiento abstractivo son actos de aprehensión. En efecto, ambos tipos de conocimiento —según mi interpretación- son actos de aprehensión en cuanto actos de formular una proposición, es decir, en cuanto actos de asentimiento (A); aunque todo acto (A) es más que un mero acto de formular una proposición.

2) La interpretación que he propuesto también coincide con la noción ockhamista de que todo conocimiento intuitivo y todo conocimiento abstractivo presupone un acto de formular una proposición. Si un conocimiento intuitivo fuera una visión — tal como Böhner lo creyó, sin especificar qué tipo de visión: una sensitiva o una intelectiva- un conocimiento intuitivo no podría ser precedido por un acto de formular una proposición debido a que - aun de manera no natural y en caso

${ }^{60}$ R. Pasnau, The Theories of Cognition in the Later Middle Ages, p. 293.

${ }^{61}$ E. Karger, “Ockham's Misunderstood Theory of Intuitive and Abstractive Cognition", p. 206.

${ }^{62}$ A. Pegis, "Some Recent Interpretations of Ockham", p. 454. 
de ilusión óptica - toda visión intelectiva es causada por una visión sensitiva y toda visión sensitiva - naturalmente y fuera de un caso de ilusión óptica- es causada por un objeto singular externo a través de alguno de los sentidos externos. ${ }^{63}$ Además, si un conocimiento intuitivo fuera un acto de visión sensible o un acto de visión intelectiva, entonces ¿cómo un conocimiento intuitivo podría verse a sí mismo en el alma sensitiva o en el alma intelectiva durante un acto reflexivo intuitivo? Esto sería imposible según Ockham.

3) La interpretación que propongo coincide con lo que Ockham expresa en un párrafo central para el estudio de la epistemología ockhamista temprana. Tal párrafo dice en latín lo siguiente:

notitia intuitiva rei est talis notitia virtute cuius potest sciri utrum res sit vel non, ita quod si res sit, statim intellectus judicat eam esse et evidenter cognoscit eam esse. (Ord. prol. I, 10-11; las cursivas son mías.)

El párrafo anterior puede traducirse así:

Un conocimiento intuitivo de una cosa es un conocimiento tal por virtud del cual se puede conocer que alguna cosa existe o no, y [por virtud del cual] al momento el intelecto juzga que la cosa existe y sabe evidentemente que existe. (Las cursivas son mías.)

El adverbio statim puede ser traducido como "al mismo tiempo", "al instante", "al momento" y no sólo como "inmediatamente" o "en seguida", según Lynn Nelson en su diccionario electrónico de latín medieval publicado por la Universidad de Notre Dame. Si traducimos statim de tal modo, un conocimiento intuitivo, según el párrafo anterior, es un acto de juicio: por él juzgamos que algo existe.

4) La interpretación que propongo permite entender que, para Ockham, un conocimiento intuitivo es una creencia verdadera en cuanto asentimiento de una proposición previamente formulada. Cada creencia verdadera intuitiva constituiría conocimiento en el momento en que fuera justificada por un acto intuitivo reflexivo. Un acto que, por hipótesis, nos permitiría saber si realmente existe el objeto singular externo al que refiere tal conocimiento intuitivo. Así también, la interpretación que propongo permite entender que un conocimiento abstractivo es una

${ }^{63}$ De manera no natural, según lo expuesto, una visión intelectiva podría ser causada por Dios. De modo natural, una visión sensitiva podría ser causada por una cualidad en el ojo. 
creencia verdadera o acto de asentimiento. Tal creencia resultaría justificada por medio de un acto reflexivo intuitivo. Un acto por el cual sabríamos que nuestra creencia verdadera está justificada en cuanto conclusión de una demostración bien formulada.

La interpretación que propongo de los conocimientos intuitivo y abstractivo sistematiza de modo preciso y económico los procesos cognoscitivos de singulares. Integra, pues, el principio de simplicidad, la máxima ockhamista mejor conocida.

\section{BIBLIOGRAFÍA}

Andrés, Teodoro de, El nominalismo de Guillermo de Ockham como filosofía del lenguaje, Gredos, Madrid, 1969.

Baños Baños, José Miguel, "Análisis funcional de los sintagmas preposicionales: Per + acusativo en latín clásico", Habis, no. 25, 1994, pp. 461-478.

Böhner, Philotheus, "The Notitia Intuitiva of Non-Existents According to William Ockham: With a Critical Study of the Text of Ockham's Reportatio and a Revised Edition of Rep. II.Q. 14-15", Traditio, no. 1, 1943, pp. 223-275.

Diccionario de la Real Academia de la Lengua Española, 22a. ed., Madrid, Real Academia de la Lengua Española, 2000.

Grellard, Christophe, Le Vocabulaire de Guillaume d'Ockham, Ellipses, París, 2005.

Karger, Elizabeth, "Ockham's Misunderstood Theory of Intuitive and Abstractive Cognition", en V. Spade, The Cambridge Companion to Ockham, Cambridge University Press, Cambridge, 1999, pp. 204-226.

Mateos, Agustín, Gramática latina, 34a. ed., Esfinge, México, 2003.

Ockham, William, Guillelmi de Ockham Opera philosophica et theologica. Opera theologica, vol. 9, Editiones Instituti Franciscani, Universitatis S. Bonaventurae, St. Bonaventure, N.Y., 1967-1986.

—, Quodlibetal Questions, trad. A.J. Freddoso y F.E. Kelley, Yale University Press, New Haven, 1991.

Panaccio, Claude, Ockham on Concepts, Ashgate, Aldershot, 2004.

Pasnau, Robert, The Theories of Cognition in the Later Middle Ages, Cambridge University Press, Cambridge, 1997.

Pegis, Anton, "Some Recent Interpretations of Ockham", Speculum, vol. 33, 1948, pp. 452-463.

Tachau, Catherine, Vision and Certitude in the Age of Ockham: Optics, Epistemology and the Foundations of Semantics, 1250-1345, E.J. Brill, Leiden, 1988. The American Heritage Dictionary, Random House, Nueva York, 2004.

Recibido el 20 de marzo de 2008; aceptado el 16 de junio de 2009. 